\title{
Interleukin-6 stimulates aerobic glycolysis by regulating PFKFB3 at early stage of colorectal cancer
}

\author{
JUN HAN $^{1 *}$, QINGYANG MENG ${ }^{1 *}$, QIULEI XI ${ }^{1}$, YONGXIAN ZHANG ${ }^{2}$, \\ QIULIN ZHUANG $^{1}$, YUSONG HAN ${ }^{1}$, YI JIANG ${ }^{1}$, QIURONG DING ${ }^{2}$ and GUOHAO WU ${ }^{1}$ \\ ${ }^{1}$ Department of General Surgery, Zhongshan Hospital, Fudan University, Shanghai; \\ ${ }^{2}$ Key Laboratory of Food Safety Research, Institute for Nutritional Sciences, Shanghai Institutes \\ for Biological Sciences, Chinese Academy of Sciences, Shanghai, P.R. China
}

Received September 5, 2015; Accepted October 13, 2015

DOI: 10.3892/ijo.2015.3225

\begin{abstract}
Chronic inflammation is a well-known etiological factor for colorectal cancer (CRC) and cancer cells are known to preferentially metabolize glucose through aerobic glycolysis. However, the connection between chronic inflammation and aerobic glycolysis in the development of CRC is largely unexplored. The present study investigated whether interleukin-6 (IL-6), a pro-inflammatory cytokine, promotes the development of CRC by regulating the aerobic glycolysis and the underlying molecular mechanisms. In colitis-associated CRC mouse, anti-IL-6 receptor antibody treatment reduced the incidence of CRC and decreased the expression of key genes in aerobic glycolysis, whereas the plasma concentrations of glucose and lactate were not affected. Consistently, IL-6 treatment stimulated aerobic glycolysis, upregulated key genes in aerobic glycolysis and promoted cell proliferation and migration in SW480 and SW1116 CRC cells. 6-Phoshofructo2-kinase/fructose-2,6-bisphosphatase-3 (PFKFB3) was the most downregulated gene by anti-IL- 6 receptor antibody in colorectal adenoma tissues. Further analysis in human samples revealed overexpression of PFKFB3 in colorectal adenoma and adenocarcinoma tissues, which was also associated with lymph node metastasis, intravascular cancer embolus and TNM stage. In addition, the effect of IL- 6 on CRC cells can be abolished by knocking down PRKFB3 through siRNA transfection. Our data suggest that chronic inflammation promotes the development of CRC by stimulating aerobic glycolysis and IL-6 is functioning, at least partly, through regulating PFKFB3 at early stage of CRC.
\end{abstract}

Correspondence to: Professor Guohao Wu, Department of General Surgery, Zhongshan Hospital, Fudan University, 180 Fenglin Road, Shanghai 200032, P.R. China

E-mail: profwugh@163.com

Key words: chronic inflammation, IL-6, aerobic glycolysis, PFKFB3, colorectal cancer

\section{Introduction}

Colorectal cancer (CRC) is the third most common cancer worldwide and its incidence has risen rapidly in Asian countries during the past few decades $(1,2)$. It has been established that environmental and hereditary factors contribute to the initiation and development of CRC, as indicated by the accumulation of mutations in oncogenes (3). Chronic inflammation is regarded as an important risk factor for the development of CRC, especially in patients with inflammatory bowel diseases (IBD) (4). Interleukin-6 (IL-6), a pro-inflammatory cytokine, has been shown to regulate cancer cell growth and thereby contribute to tumor promotion and progression (5). IL-6 has been demonstrated to be associated with an unfavorable prognosis in patients with various types of cancers including both sporadic and colitis-associated CRC (6). However, despite clear evidence implicating IL-6 in causation of CRC, molecular mechanisms underlying this phenomenon are still incompletely understood.

Cancer cells preferentially use glucose by aerobic glycolysis, which is characterized by increased glycolysis and lactate production regardless of oxygen availability. This phenomenon, termed as the Warburg effect, is a metabolic adaptation that promotes the proliferation of cancer cells and is added as an emerging hallmark of cancer (7-9). Tumor hypoxia was thought to be a major contributor in the switch to aerobic glycolysis and hypoxia inducible factor- $1 \alpha$ (HIF-1 $\alpha$ ) was thought to play an important role in the increased aerobic glycolysis in cancer cells (10). However, cancer cells exhibit aerobic glycolysis even though tumor cells are exposed to oxygen during tumorigenesis (11). Thus, what triggers the switch from oxidative phosphorylation to aerobic glycolysis remains unclear.

Fructose-2,6-bisphosphate (F2,6-BP) is a powerful allosteric activator of phospho-fructokinase 1 (PFK-1), the enzyme that controls one of the most critical steps of glycolysis (12). F2,6-BP is synthesised by the family of 6-phosphofructo2-kinase/2,6-bisphosphatase (PFKFB) bifunctional enzymes. PFKFB3 is the most efficient isoform of this family and is overexpressed in various types of cancers including colon cancer (13). PFKFB3 was demonstrated to be a hypoxia inducible gene that was stimulated through HIF-1 interaction with 
the consensus hypoxia response element site in its promoter region (14). However, the relationship between PFKFB3 and chronic inflammation is rarely reported. In addition, what is the function of PFKFB3 during the initiation and development of $\mathrm{CRC}$ is still unclear.

In the present study, we investigated whether IL-6 promotes the development of CRC by regulating the aerobic glycolysis and the underlying molecular mechanisms. We found that anti-IL-6 receptor antibody decreased the expression of key genes involved in aerobic glycolysis, whereas IL-6 treatment upregulated PRKFB3 expression and promoted glycolysis in CRC cells. Further analysis in human samples revealed higher PRKFB3 expression in colorectal adenoma and adenocarcinoma tissues, which was also associated with lymph node metastasis, intravascular cancer embolus and TNM stage in sporadic CRC patients. Knockdown of PFKFB3 in CRC cells also abolished IL-6 stimulated cell proliferation and migration. Overall, our results indicate that chronic inflammation promotes the initiation and development of CRC and IL-6 is functioning, at least partly, through regulating PFKFB3 at early stage of CRC.

\section{Materials and methods}

Patients specimens. Eighty-seven sporadic and 13 colitisassociated CRC tumor tissues and their matching adjacent non-malignant tissues were collected during surgery from patients in Zhongshan Hospital of Fudan University. Colorectal pathological sections of patients who were diagnosed as chronic inflammation $(n=18)$, adenoma $(n=23)$ and adenocarcinoma $(n=26)$ were collected from the Pathology Department of Zhongshan Hospital of Fudan University. The patients recruited to the present study had not received chemotherapy or radiotherapy before surgery. Written informed consents were obtained from all the patients and permission for this study was obtained from the ethics committee of Zhongshan Hospital of Fudan University. Fresh specimens were immediately frozen in liquid nitrogen and stored at $-80^{\circ} \mathrm{C}$ until further analysis.

Mouse and colitis-associated CRC model. The colitis-associated CRC mouse model was induced in Balb/c male mice (6-8 weeks of age) purchased from the Shanghai Laboratory Animal Center, Chinese Academy of Sciences. Mice were acclimatized to the environment for a week before the study started. All animal manipulations were carried out according to the guidelines of regulations for the use of experimental animals of the Chinese Academy of Science. All efforts were made to minimize animal suffering. Mice were divided into 3 groups: normal saline control group (NS group, $n=16$ ), mouse monoclonal IgG (eBiosciences) control group (IgG group, $\mathrm{n}=16$ ), mouse monoclonal anti-IL-6 receptor antibody (eBiosciences) treated group (IL-6R Ab group, $n=16$ ). Colitis-associated CRC model protocol is shown in Fig. 1A as previously described (15). Mice were peritoneal injected with azoxymethane (AOM, $10 \mathrm{mg} / \mathrm{kg}$; Sigma) once at the beginning of the first week. Then, mice were treated with $2 \%$ (w/v) dextran sulfate sodium (DSS; Sigma) in the drinking water for a week, followed by 2 weeks of regular water for 3 cycles. In IL-6R Ab treated group, each mouse was peritoneally injected with $10 \mu \mathrm{g}$ monoclonal
anti-IL-6 receptor antibody diluted in $200 \mu 1$ normal saline every 2 days. IgG diluted $(10 \mu \mathrm{g})$ in $200 \mu \mathrm{l}$ normal saline and $200 \mu 1$ normal saline were peritoneally injected into mice in the IgG and NS groups, respectively. Eight mice in each group were sacrificed at the end of week 6 to investigate the effect of anti-IL- 6 receptor antibody on the early stage of CRC, and 8 mice in each group were sacrificed at the end of week 9 to evaluate the effect of anti-IL-6 receptor antibody on the CRC. Blood sample of each mouse was collected in standard serum tubes and was immediately centrifuged at $2400 \mathrm{x}$ g for $10 \mathrm{~min}$. The plasma was removed and preserved at $-80^{\circ} \mathrm{C}$ until further analysis. Large bowel (from the ileocecal junction to the anal verge) of each mouse was collected and cut open longitudinally along the main axis. Visible tumors were cut from the mucosa and the numbers of tumors were recorded. The length and the width of each tumor were measured by a digital micro-ruler. The tumor size was calculated by multiplying the length and the width. All tumors were cut into halves. One half was prepared for histopathological analysis and immunohistochemistry, and the other half was prepared for quantitative real-time PCR (RT-qPCR) analysis and western blot analysis.

Histopathological analysis. One half of each tumor was fixed in $4 \%$ formalin and embedded in paraffin. Tissue was sectioned at $4-\mu \mathrm{m}$ thickness and stained with hematoxylin and eosin (H\&E). The slides were read by two pathologists to determine the tumor to be colorectal adenoma or adenocarcinoma.

Cell culture. Human SW480 and SW1116 CRC cells were obtained from the American Type Culture Collection (ATCC; Manassas, VA, USA) and cultured at $37^{\circ} \mathrm{C}$ in Leibovitz's L15 medium (Gibco, Carlsbad, CA, USA) supplemented with $10 \%$ fetal bovine serum (FBS; Sigma), 100 units/ml of penicillinstreptomycin (Invitrogen). For inflammatory stimulation, $20 \mathrm{ng} / \mathrm{ml}$ recombinant human IL-6 (PeproTech, Inc., Rocky Hill, USA) was used to treat cells.

Small interfering RNA (siRNA) transfection. Transfection of siRNAs was carried out using Lipofectamine RNAiMAX (Invitrogen) following the manufacturer's instructions. Cells were $40-60 \%$ confluent at the time of the transfection. The following siRNAs for PFKFB3 were used: PFKFB3 siRNA1 (Invitrogen; \#PFKFB3HSS107862) and PFKFB3 siRNA2 (Invitrogen; \#PFKFB3HSS107861). The universal control siRNA (Invitrogen; \#12935-112) was used as control. Cells were incubated at $37^{\circ} \mathrm{C}$ for $48 \mathrm{~h}$ before harvest. For inflammatory stimulation, cells were treated with $20 \mathrm{ng} / \mathrm{ml}$ recombinant human IL-6 for $24 \mathrm{~h}$ prior to harvest.

Measurement of glucose and lactate. Mouse plasma concentration of glucose was determined using a glucose assay kit (Sigma) and plasma concentration of lactate was determined using the lactate assay kit (BioVision). Glucose consumption and lactate production in CRC cells were analyzed as previously described (16). Glucose and lactate levels in the culture medium were also determined by using the glucose assay kit and lactate assay kit, respectively.

Immunohistochemistry (IHC). IHC of human and mouse colorectal tissues were performed as previously described (17). 


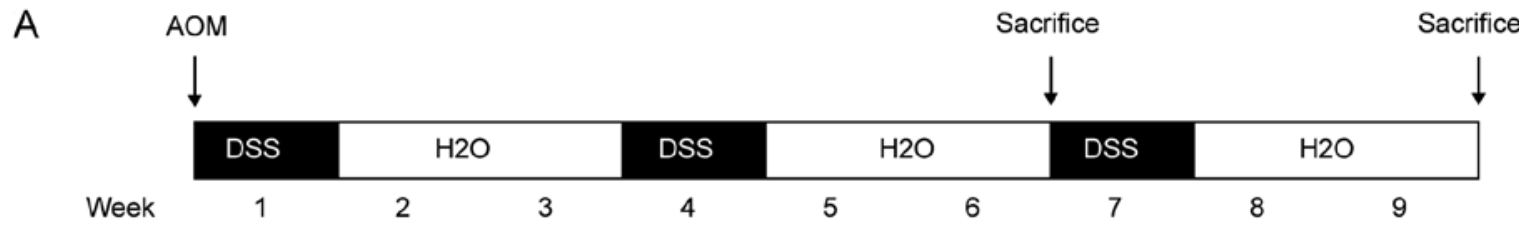

B
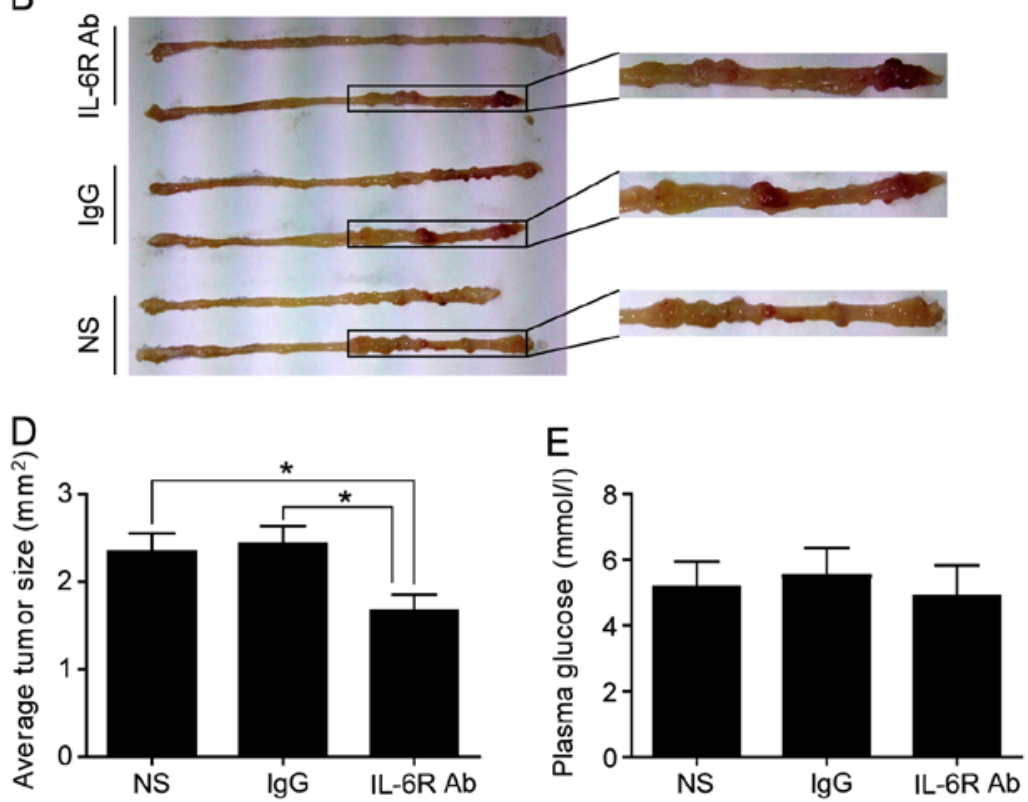
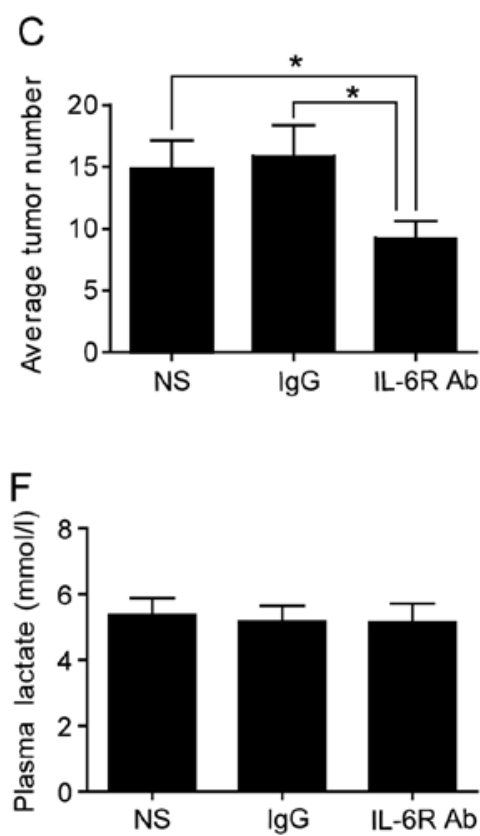

Figure 1. The effects of anti-IL-6 receptor antibody on AOM and DSS colitis-related CRC mouse model. (A) Schematic time schedule of the AOM and DSS mouse model. Mice were treated with a single AOM injection at the beginning of the first week, followed by 3 cycles of DSS in drinking water for a week and regular water for 2 weeks. Anti-IL-6 receptor antibody, IgG and normal saline were injected every 2 days in the treated and control groups, respectively. Eight mice in each group were sacrificed at the end of week 6 and week 9, respectively. (B-D) Fewer and smaller macroscopic neoplasms were observed in treated group than control groups. (E and F) Plasma concentrations of glucose and lactate were not significantly different between treated group and control groups. ${ }^{*} \mathrm{P}<0.05$.

Briefly, slides were dehydrated in xylene and graded alcohols. Antigen retrieval was performed with $0.01 \mathrm{M}$ citrate buffer at $\mathrm{pH} 6.0$ at $95^{\circ} \mathrm{C}$ for $20 \mathrm{~min}$. Slides were incubated with diluted primary antibodies [anti-lactate dehydrogenase isoform A (LDHA), 1:100 dilution; anti-PKKFB3, 1:150 dilution; anti-glucose transporter 1 (GLUT1), 1:250 dilution; anti-pyruvate kinase isoform M2 (PKM2), 1:150 dilution; and anti-hexokinase 2 (HK2), 1:400 dilution] for $12 \mathrm{~h}$. Then slides were incubated with biotinylated secondary antibody for $1 \mathrm{~h}$, peroxidase-labeled streptavidin for $15 \mathrm{~min}$, and diaminobenzidine and hydrogen peroxide chromogen substrate plus diaminobenzidine enhancer (Dako) for $10 \mathrm{~min}$, followed by counter staining with Mayer's hematoxylin.

$R T-q P C R$ analysis. Total RNAs were isolated from the cells and tissues with TRIzol (Invitrogen) and cDNAs were synthesized from $1 \mu \mathrm{g}$ total RNA using the cDNA Synthesis kit (Takara) following the manufacturer's protocol. RT-qPCR reactions were performed in StepOnePlus Real-Time system (Applied Biosystems) and the expression levels of target genes relative to $\beta$-actin were determined by a SYBR-Green-based comparative $\mathrm{Ct}$ method $\left(2^{-\Delta \Delta \mathrm{Ct}}\right)$. Experiments were repeated at least 3 times. Primers used are listed in Table I.

Western blot analysis. Total proteins were extracted from cells or tissues and quantified by the BCA method (Bio-Rad
Laboratories). The western blot analysis was performed as previously described (17). For detection of PFKFB3, we used a rabbit monoclonal anti-PFKFB3 antibody (1:6,000 dilution; Abcam). Tubulin (1:2,000 dilution; Sigma) expression was used as an endogenous control.

MTT cell proliferation assay. The proliferation rates of SW480 and SW1116 cells transfected with PFKFB3 siRNAs and control siRNA with or without IL-6 stimulation were measured by MTT assay. Cells were divided into 4 groups: siRNA1 with IL-6 group, siRNA2 with IL-6 group, control siRNA with IL-6 group, and control siRNA without IL-6 group. Briefly, cells were transfected with PFKFB3 siRNAs for $48 \mathrm{~h}$. Cells were then seeded at a density of 5,000 cells in $200 \mu \mathrm{l}$ of medium with or without IL-6 in a 96-well cell culture plate and incubated for 24,48 and $72 \mathrm{~h}$, respectively. Cells were then treated with $20 \mu \mathrm{l}$ MTT ( $5 \mathrm{mg} / \mathrm{ml}$; Sigma) and incubated for $4 \mathrm{~h}$. The generated formazan was dissolved in $150 \mu \mathrm{l}$ of dimethylsulfoxide (DMSO) after the medium was discarded. Cell viability was determined in a microplate reader at $490 \mathrm{~nm}$ with subtraction of the baseline reading.

Transwell cell migration assay. Migration ability of CRC cells was determined with a Transwell chamber (Corning Incorporated). Briefly, cells were treated and divided into 4 groups as described above. Cells were resuspended in $100 \mu \mathrm{l}$ of 
Table I. Primer sequences of target genes and $\beta$-actin.

\begin{tabular}{|c|c|c|c|}
\hline Species & Genes & Sense/antisense & Sequence \\
\hline Human & GLUT1 & $\begin{array}{l}\text { Sense } \\
\text { Antisense }\end{array}$ & $\begin{array}{l}\text { TTCACTGTCGTGTCGCTGTTTG } \\
\text { TCACACTTGGGAATCAGCCCC }\end{array}$ \\
\hline Human & HK2 & $\begin{array}{l}\text { Sense } \\
\text { Antisense }\end{array}$ & $\begin{array}{l}\text { CAAAGTGACAGTGGGTGTGG } \\
\text { GCCAGGTCCTTCACTGTCTC }\end{array}$ \\
\hline Human & PKM2 & $\begin{array}{l}\text { Sense } \\
\text { Antisense }\end{array}$ & $\begin{array}{l}\text { CCACTTGCAATTATTTGAGGAA } \\
\text { GTGAGCAGACCTGCCAGACT }\end{array}$ \\
\hline Human & PFKFB3 & $\begin{array}{l}\text { Sense } \\
\text { Antisense }\end{array}$ & $\begin{array}{l}\text { CCTCACTCGCAGCCACTTCT } \\
\text { CAGTTCCTACTCAATTCCAA }\end{array}$ \\
\hline Human & LDHA & $\begin{array}{l}\text { Sense } \\
\text { Antisense }\end{array}$ & $\begin{array}{l}\text { TTGACCTACGTGGCTTGGAAG } \\
\text { GGTAACGGAATCGGGCTGAAT }\end{array}$ \\
\hline Mouse & GLUT1 & $\begin{array}{l}\text { Sense } \\
\text { Antisense }\end{array}$ & $\begin{array}{l}\text { TATGGTAAAGAGCCGCCTAA } \\
\text { GCACTGCCAGATTCAAACA }\end{array}$ \\
\hline Mouse & HK2 & $\begin{array}{l}\text { Sense } \\
\text { Antisense }\end{array}$ & $\begin{array}{l}\text { GTGAGCCATCGTGGTTAAGC } \\
\text { GCGAGGCGATCATCTTGTTG }\end{array}$ \\
\hline Mouse & PKM2 & $\begin{array}{l}\text { Sense } \\
\text { Antisense }\end{array}$ & $\begin{array}{l}\text { TGTCTGGAGAAACAGCCAAG } \\
\text { TCCTCGAATAGCTGCAAGTG }\end{array}$ \\
\hline Mouse & PFKFB3 & $\begin{array}{l}\text { Sense } \\
\text { Antisense }\end{array}$ & $\begin{array}{l}\text { AGGTCGGCATGTTGAAGAGT } \\
\text { AGAGAACAGAGCGTAGGAAG }\end{array}$ \\
\hline Mouse & LDHA & $\begin{array}{l}\text { Sense } \\
\text { Antisense }\end{array}$ & $\begin{array}{l}\text { TGTCTCCAGCAAAGACTACTGT } \\
\text { GACTGTACTTGACAATGTTGGGA }\end{array}$ \\
\hline Human/mouse & $\beta$-actin & $\begin{array}{l}\text { Sense } \\
\text { Antisense }\end{array}$ & $\begin{array}{l}\text { CACGATGGAGGGGCCGGACTCATC } \\
\text { TAAAGACCTCTATGCCAACACAGT }\end{array}$ \\
\hline
\end{tabular}

GLUT1, glucose transporter-1; HK2, hexokinase-2; PKM2, pyruvate kinase isoform M2; PKKFB3, 6-phoshofructo-2-kinase/fructose2,6-bisphosphatase-3; LDHA, lactate dehydrogenase isoform-A.

FBS-free medium and placed in the upper chamber, whereas the lower chamber contained $500 \mu \mathrm{l}$ of $10 \%$ FBS medium with or without IL-6. After $24 \mathrm{~h}$, cells remaining on the upper side of the membrane were cleared, and the migrated cells on the lower side of the membrane were fixed with paraformaldehyde (PFA) and stained with $0.1 \%$ crystal violet. The number of cells from 5 random microscopic fields was quantified for each group.

Statistical analysis. All results are presented as the means \pm standard error of the mean (SEM) unless indicated otherwise. The correlation of PFKFB3 expression with the clinicopathological factors in sporadic CRC patients was analyzed using the $\chi^{2}$ test. The difference between two groups was analyzed using the Student's t-test. All statistical analyses were performed using the GraphPad Prism 5.0 software or Stata version 11.0. $\mathrm{P}<0.05$ was considered statistically significant.

\section{Results}

Anti-IL-6 receptor antibody reduces the incidence of colitisrelated $C R C$, but does not affect the plasma concentrations of glucose and lactate. The AOM and DSS colitis-associated
CRC mouse model was induced as shown in Fig. 1A. The mice were treated with anti-IL-6 receptor antibody to block IL-6 mediated chronic inflammation or normal saline and IgG as control. Eight mice in each group were sacrificed at the end of week 9 to investigate the effect of anti-IL-6 receptor antibody on the development of CRC. Mice in treated group developed fewer and smaller macroscopic colorectal tumors (Fig. 1B-D). Plasma concentrations of glucose and lactate were not significantly different between the treated group and the control groups (Fig. 1E and F). These data suggested that antiIL-6 receptor antibody treatment could reduce the incidence of colorectal tumors, with no significant effect on plasma concentrations of glucose and lactate.

Anti-IL-6 receptor antibody downregulates the key genes involved in aerobic glycolysis in colorectal adenocarcinoma tissues. To clarify the potential mechanism by which anti-IL-6 receptor antibody inhibits tumor formation in the colitis-associated CRC mouse model, and to further investigate whether this inhibition is through regulation of aerobic glycolysis, we examined the expression of a number of key genes involved in aerobic glycolysis (including GLUT1, HK2, PFKFB3, PKM2 and and LDHA) in colorectal adenocarcinoma tissues. 
A

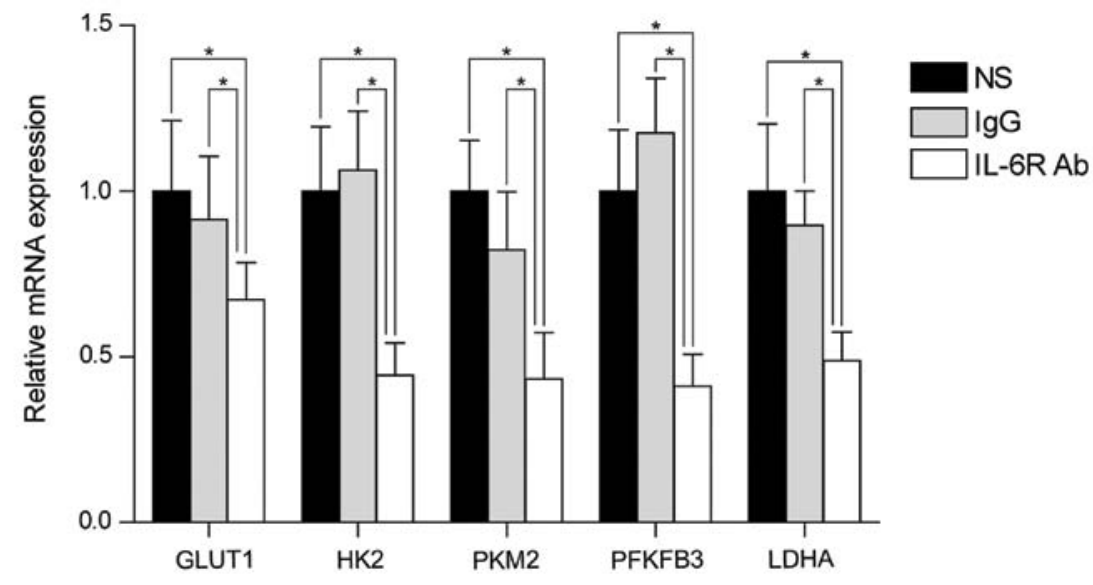

B

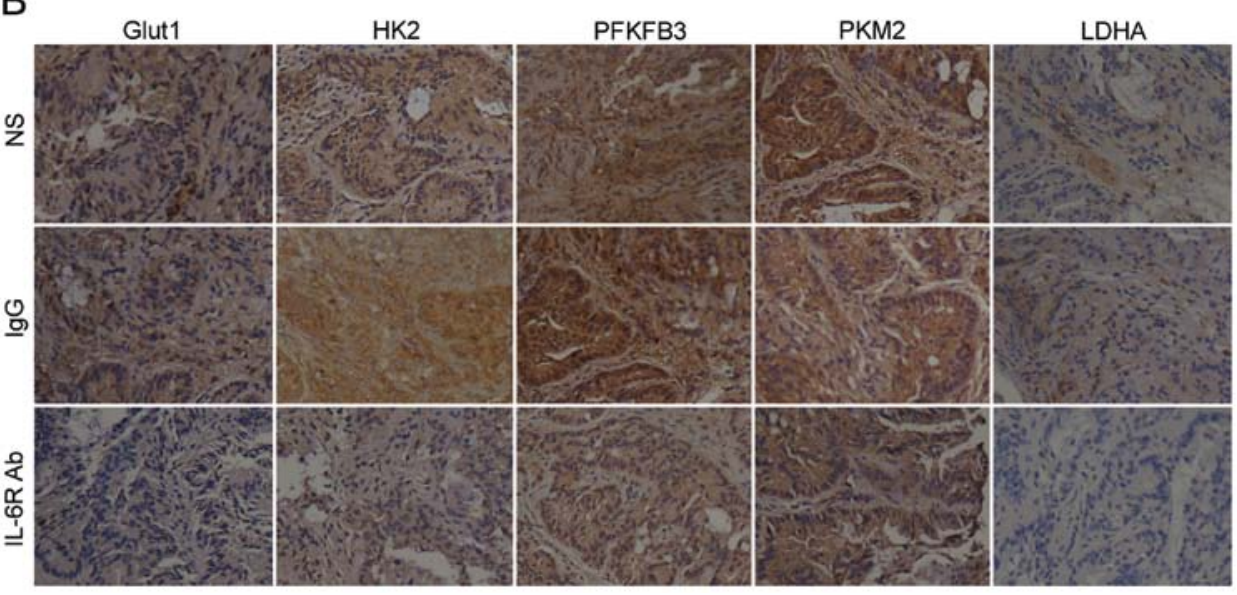

Figure 2. The effects of anti-IL-6 receptor antibody on 5 key genes involved in aerobic glycolysis in mouse colorectal adenocarcinomas tissues. (A) RT-qPCR analysis showed the mRNA levels of these genes were downregulated in the treated group. (B) Immunohistochemistry analysis showed the protein levels of these genes were also downregulated in the treated group (magnification, $\mathrm{x} 400$ ); ${ }^{*} \mathrm{P}<0.05$.
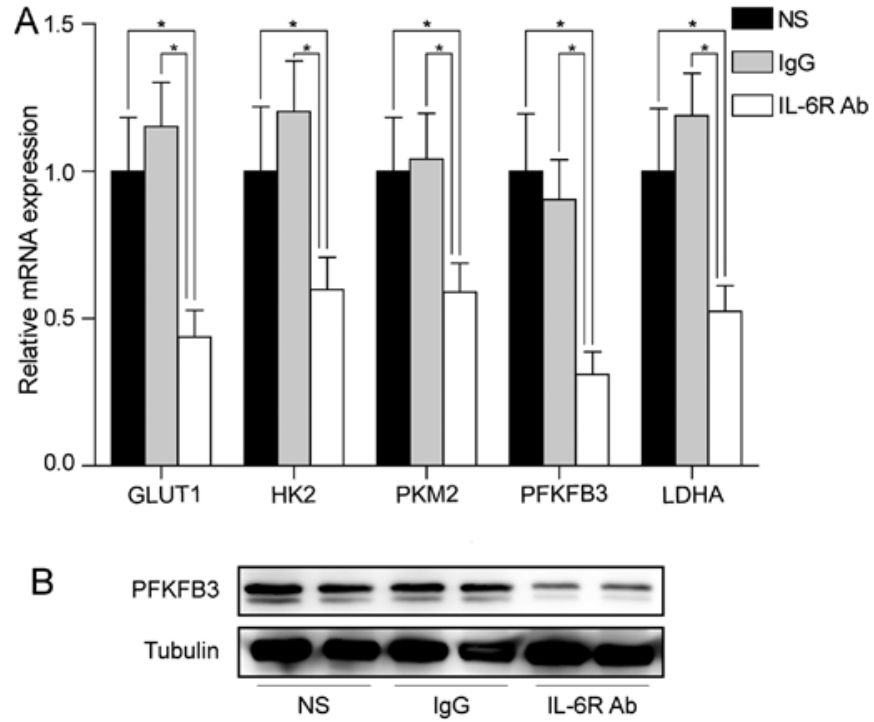

Figure 3. The effects of anti-IL-6 receptor antibody on 5 key genes involved in aerobic glycolysis in mouse colorectal adenomas tissues. (A) RT-qPCR analysis showed the mRNA levels of these genes were downregulated in the treated group, especially the PFKFB3. (B) Western blot analysis confirmed the downregulation of $\mathrm{PFKFB} 3$ protein in the treated group; ${ }^{*} \mathrm{P}<0.05$.

RT-qPCR analysis showed that these genes were downregulated after treatment with anti-IL-6 receptor antibody
(Fig. 2A). In line with the mRNA levels, the protein levels of these genes were also downregulated after anti-IL-6 receptor antibody treatment (Fig. 2B). These data demonstrated that anti-IL-6 receptor antibody might inhibit colorectal adenocarcinoma formation through regulation of aerobic glycolysis.

Anti-IL-6 receptor antibody downregulates the key genes involved in aerobic glycolysis in colorectal adenoma tissues. We next sought to investigate whether the change in aerobic glycolysis genes occurred already at the early stage of CRC. Eight mice in each group were sacrificed at the end of week 6 . We examined the mRNA levels of key genes involved in aerobic glycolysis in colorectal adenoma tissues. Similar mRNA levels of downregulation of these genes were observed in colorectal adenoma tissues after treatment with anti-IL-6 receptor antibody (Fig. 3A). Notably, PFKFB3 was the most downregulated gene by anti-IL- 6 receptor antibody in colorectal adenoma tissues, suggesting that PFKFB3 might play an important role at early stage of CRC. Western blot analysis also confirmed the downregulation of PFKFB3 protein in the treated group (Fig. 3B).

PFKFB3 is overexpressed in human sporadic and colitisassociated CRC tumor tissues and colorectal adenoma tissues. Given PFKFB3 was the most influenced gene at early stage of CRC in colitis-associated CRC model, we analyzed 
A

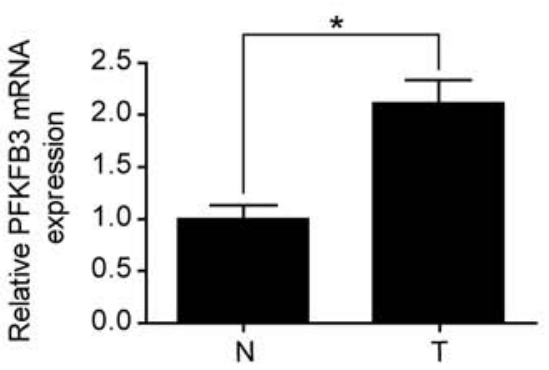

B

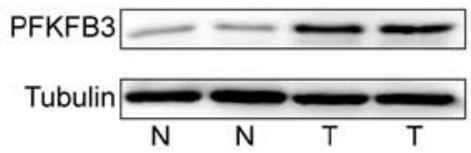

C

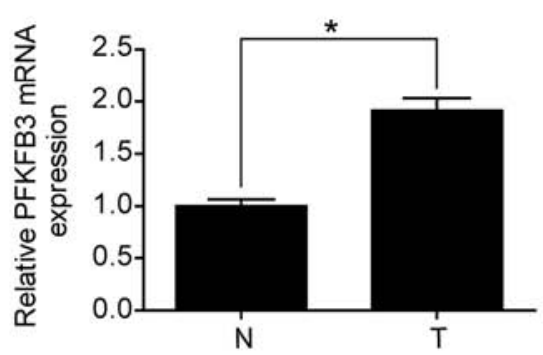

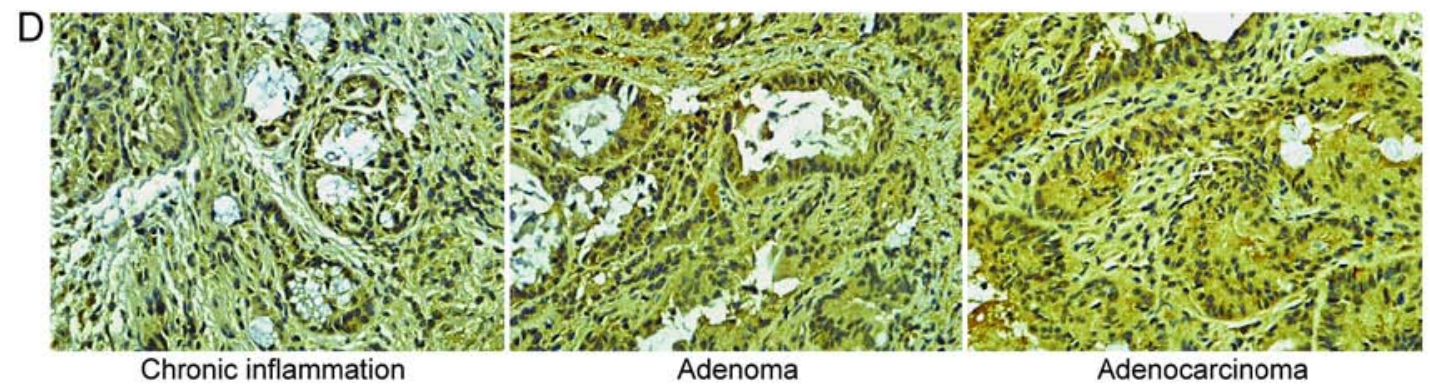

Figure 4. Expression of PFKFB3 in human sporadic and colitis-associated CRC tissues and colorectal adenoma tissues. (A) RT-qPCR analysis showed PFKFB3 mRNA was highly expressed in tumor tissues compared to adjacent non-malignant tissues in colitis-associated CRC patients. (B) Western blot analysis also confirmed the overexpression of PFKFB3 protein in tumor tissues of colitis-associated CRC patients. (C) RT-qPCR analysis showed the PFKFB3 mRNA was highly expressed in tumor tissues compared to adjacent non-malignant tissues in sporadic CRC patients. (D) Immunohistochemistry analysis showed PFKFB3 protein was highly expressed in colorectal adenoma and adenocarcinoma tissues compared with chronic inflammation tissues, but no significant difference was observed between adenoma and adenocarcinoma tissues (magnification, 400). T, tumor tissues; $\mathrm{N}$, adjacent non-malignant tissues; ${ }^{*} \mathrm{P}<0.05$.
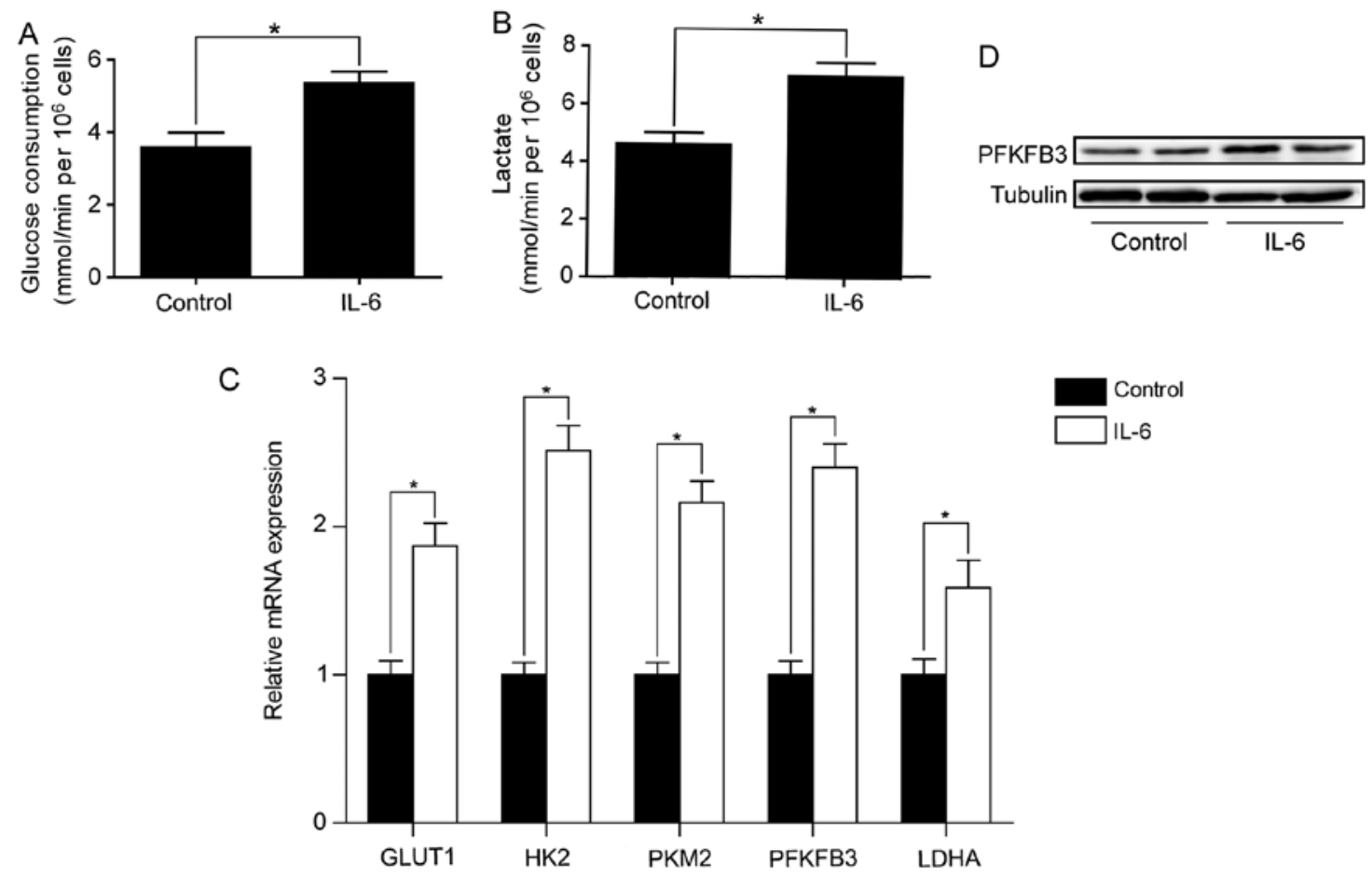

Figure 5. IL-6 promotes aerobic glycolysis and upregulates key genes in aerobic glycolysis in SW480 cells. (A and B) IL-6 increased the rate of glucose consumption and lactate production in SW480 cells. (C) RT-qPCR analysis showed the mRNA levels of 5 key genes in aerobic glycolysis were upregulated by IL-6 treatment. (D) Western blot analysis confirmed the upregulation of PFKFB3 protein by IL- 6 treatment. * $\mathrm{P}<0.05$.

whether PFKFB3 was differentially expressed in different human colorectal tissues. Firstly, we examined the expression of PFKFB3 in 13 colitis-associated CRC patients. The results revealed that PFKFB3 mRNA level was significantly increased in tumor tissues compared to adjacent non-malignant tissues (Fig. 4A). Overexpression of PFKFB3 protein in tumor tissues was also confirmed by western blot analysis (Fig. 4B). Secondly, we examined the expression of PFKFB3 
A

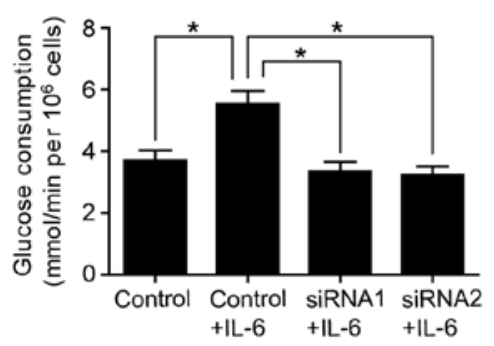

B

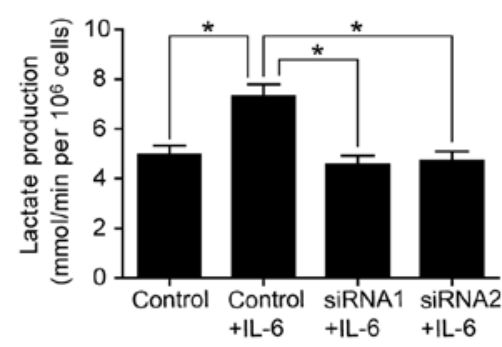

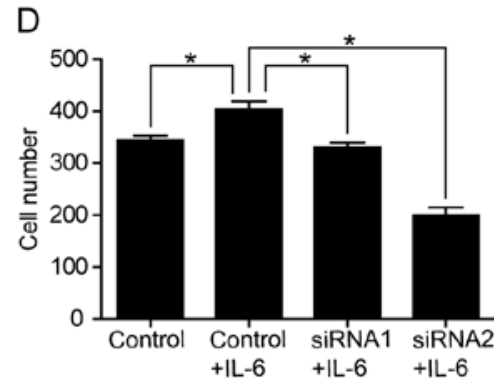

C

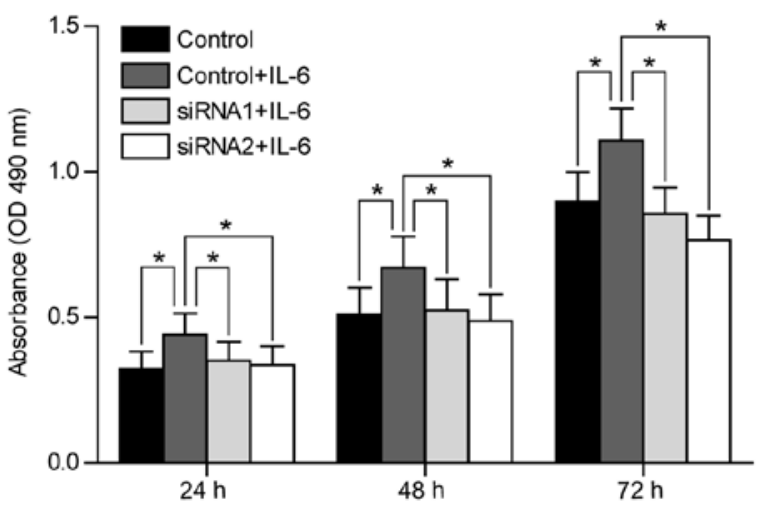

$\mathrm{E}$

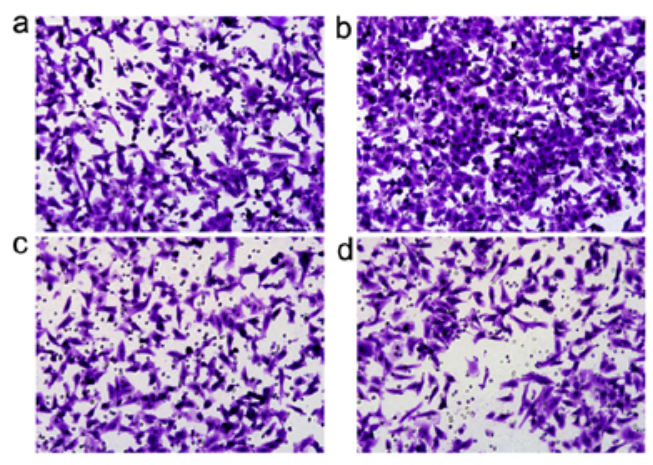

Figure 6. Knockdown of PFKFB3 inhibits cell proliferation and migration stimulated by IL-6 treatment in SW480 cells. (A and B) The increased rate of glucose consumption and lactate production by IL- 6 treatment was abolished by PFKFB3 siRNAs. (C) MTT cell proliferation assay showed that the enhanced cell proliferation ability stimulated by IL- 6 treatment was abolished by PFKFB3 siRNAs. (D and E) Transwell cell migration assay showed that the enhanced cell migration ability stimulated by IL-6 treatment was abolished by PFKFB3 siRNAs (magnification, x100). a, control siRNA group; b, control siRNA + IL- 6 group; c, siRNA1 + IL-6 group; d, siRNA2 + IL-6 group; ${ }^{*} \mathrm{P}<0.05$.

in 87 sporadic CRC patients and analyzed its correlation with the clinicopathological factors. As shown in Fig. 4C, RT-qPCR results validated the overexpression of PFKFB3 in tumor tissues compared to adjacent non-malignant tissues in sporadic CRC patients. Furthermore, correlation analysis indicated that PFKFB3 mRNA high expression was significantly associated with lymph node metastasis, intravascular cancer embolus, and TNM stage in 87 sporadic CRC patients (Table II).

To clarify whether PFKFB3 expression increases gradually in colorectal tissues from chronic inflammation to adenoma and adenocarcinoma, we examined the PFKFB3 expression in colorectal pathological sections of patients who were diagnosed as colorectal chronic inflammation, adenoma and adenocarcinoma. Notably, we found that PFKFB3 was highly expressed in colorectal adenoma and adenocarcinoma tissues compared with chronic inflammation tissues, but no significant difference was observed between adenoma and adenocarcinoma tissues (Fig. 4D). The results indicated that PFKFB3 might play an important role starting at the colorectal adenoma stage.

IL-6 promotes glycolysis and upregulates PFKFB3 in SW480 and SW1116 cells. Our data thus far suggested that IL-6 promotes the initiation and progression of CRC by regulating aerobic glycolysis. We next sought to address the puzzle that plasma concentrations of glucose and lactate in mice were not significantly affected after treatment with anti-IL-6 receptor antibody. We reasoned that IL-6 might influence the local concentrations of glucose and lactate but not the concentrations in whole blood. To this end, we first examined the effect of IL-6 on aerobic glycolysis in SW480 and SW1116 cells. As shown in Fig. 5A and B, IL-6 treatment increased the rates of glucose consumption and lactate production in SW480 cells. We also examined the effect of IL- 6 on the expression of key genes involved in aerobic glycolysis. RT-qPCR analysis showed that these genes were significantly upregulated by IL- 6 treatment in SW480 cells (Fig. 5C). Among these genes, PFKFB3 was increased by 2.4-fold. In line with Q-PCR result, western blot analysis confirmed the increased PFKFB3 protein level after IL-6 treatment in SW480 cells (Fig. 5D). We also assessed the effect of IL-6 on aerobic glycolysis and PFKFB3 expression in a second CRC cell line (SW1116) and found enhanced aerobic glycolysis as well as increased PFKFB3 expression by IL-6 treatment (data not shown).

Knockdown of PFKFB3 inhibits IL-6 stimulated cell proliferation and migration. Given that IL-6 upregulated PFKFB3 expression in CRC cells, and overexpression of PFKFB3 was associated with lymph node metastasis and TNM stage in CRC patients, we hypothesized that IL-6 might stimulate CRC cell proliferation and migration through regulating PFKFB3. To test this hypothesis, we examined whether the increased rates of glucose consumption and lactate production by IL-6 treatment could be abolished by knocking down PFKFB3. Indeed, we found that PRKFB3 siRNAs reversed the increase of glucose consumption and lactate production by IL-6 treatment in SW480 cells (Fig. 6A and B). Consistently, PFKFB3 siRNAs abolished the enhanced cell proliferation and migration stimulated by IL-6 in SW480 cells (Fig. 6C-E), suggesting 


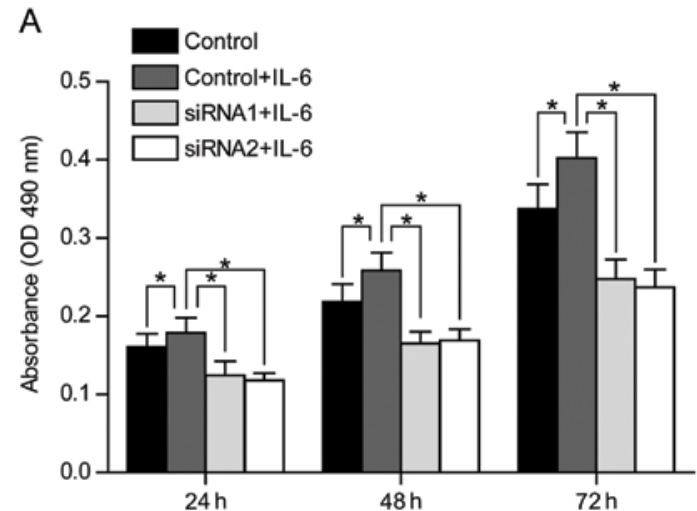

B
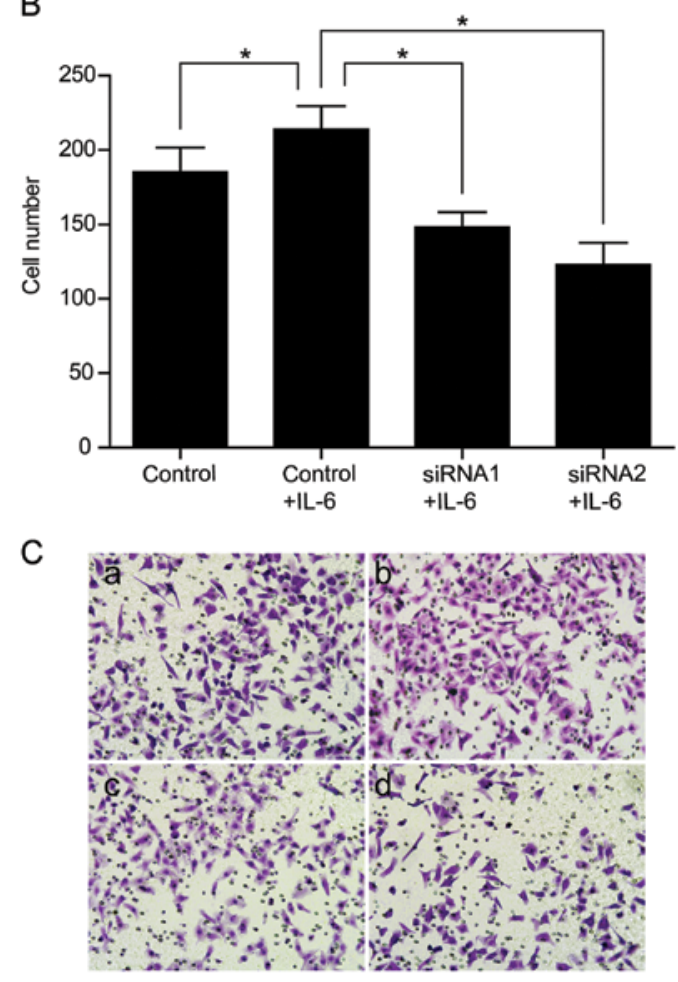

Figure 7. Knockdown of PFKFB3 inhibits cell proliferation and migration stimulated by IL-6 treatment in SW1116 cells. (A) MTT cell proliferation assay showed that the enhanced proliferation ability stimulated by IL-6 treatment was abolished by PFKFB3 siRNAs. (B and C) Transwell cell migration assay showed that the enhanced migration ability stimulated by IL-6 treatment was abolished by PFKFB3 siRNAs (magnification, $x 100$ ). a, control siRNA group; b, control siRNA + IL-6 group; c, siRNA1 + IL-6 group; d, siRNA2 + IL-6 group. ${ }^{*} \mathrm{P}<0.05$.

that IL-6 functions through regulating PFKFB3. We also confirmed the inhibition effect of PFKFB3 siRNAs on cell proliferation and migration in SW1116 cells (Fig. 7).

\section{Discussion}

Chronic inflammation is a well-known risk factor for CRC and the aerobic glycolysis of cancer cells is also well documented $(7,18)$. However, whether chronic inflammation is attributed to tumor initiation and progression of CRC through regulating the aerobic glycolysis remains largely unknown. In this study, we found that blocking of IL-6 function significantly inhibited the initiation and progression of colitis-associated $\mathrm{CRC}$ and decreased the expression of key genes involved in
Table II. Clinicopathological correlation of PFKFB3 expression in sporadic colorectal cancer patients.

\begin{tabular}{|c|c|c|c|c|}
\hline \multirow[b]{2}{*}{ Features } & \multicolumn{2}{|c|}{ PFKFB3 } & \multirow[b]{2}{*}{$\chi^{2}$} & \multirow[b]{2}{*}{ P-value } \\
\hline & Low & High $^{\mathrm{a}}$ & & \\
\hline All cases & 43 & 44 & & \\
\hline Gender & & & 0.3118 & 0.577 \\
\hline Male & 26 & 24 & & \\
\hline Female & 17 & 20 & & \\
\hline Age (years) & & & 0.0975 & 0.755 \\
\hline$>65$ & 23 & 25 & & \\
\hline$\leq 65$ & 20 & 19 & & \\
\hline $\begin{array}{l}\text { Intravascular } \\
\text { cancer embolus }\end{array}$ & & & 4.2548 & 0.039 \\
\hline Present & 5 & 13 & & \\
\hline Absent & 38 & 31 & & \\
\hline Perineuronal invasion & & & 1.8856 & 0.170 \\
\hline Present & 9 & 15 & & \\
\hline Absent & 34 & 29 & & \\
\hline Tumor size $(\mathrm{cm})$ & & & 2.2123 & 0.137 \\
\hline$>4$ & 12 & 19 & & \\
\hline$\leq 4$ & 31 & 25 & & \\
\hline T stage & & & 1.046 & 0.79 \\
\hline $\mathrm{T} 1$ & 3 & 2 & & \\
\hline $\mathrm{T} 2$ & 11 & 9 & & \\
\hline $\mathrm{T} 3$ & 11 & 10 & & \\
\hline $\mathrm{T} 4$ & 18 & 23 & & \\
\hline N stage & & & 7.622 & 0.033 \\
\hline NO & 18 & 8 & & \\
\hline N1 & 13 & 14 & & \\
\hline $\mathrm{N} 2$ & 12 & 22 & & \\
\hline M stage & & & 2.591 & 0.107 \\
\hline M0 & 38 & 33 & & \\
\hline M1 & 5 & 11 & & \\
\hline \multicolumn{5}{|l|}{ Tumor stage } \\
\hline $\mathrm{I}$ & 5 & 3 & 8.4802 & 0.037 \\
\hline II & 16 & 6 & & \\
\hline III & 17 & 24 & & \\
\hline IV & 5 & 11 & & \\
\hline
\end{tabular}

${ }^{a}$ The median expression level was used as the cut-off. Low expression of PFKFB3 in 43 patients was classified as values below the 50th percentile. High PFKFB3 expression in 44 patients was classified as

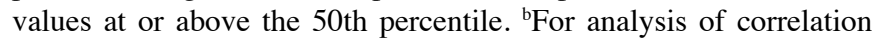
between PFKFB3 and clinicopathological features, the $\chi^{2}$ test was used. Results were considered statistically significant at $\mathrm{P}<0.05$.

aerobic glycolysis (especially the PFKFB3) even at early stage of CRC. PFKFB3 was demonstrated to be overexpressed in human colorectal adenoma and adenocarcinoma tissues, and the high expression of PFKFB3 mRNA was associated with lymph node metastasis, intravascular cancer embolus and TNM 
stage in sporadic CRC patients. IL-6 was also demonstrated to accelerate aerobic glycolysis and upregulate key genes involved in aerobic glycolysis in vitro, whereas knockdown of PFKFB3 abolished the enhanced proliferation and migration abilities stimulated by IL- 6 in CRC cells. These data indicate that IL-6 might promote the initiation and progression of CRC by regulating PFKFB3 at early stage of CRC.

AOM-induced CRC mouse model is widely used and can resemble human $\mathrm{CRC}$ in many respects including the molecular level $(19,20)$. AOM and DSS-induced CRC mouse model is a model for colitis-associated tumor development, which is particularly applicable when the study focuses on tumor progression driven by chronic colitis (15). Balb/c mice were reported to be extremely sensitive to AOM and DSS-induced colitis-associated CRC (21). In the presenr study, using the combined treatment of one exposure of AOM and 3 cycles of DSS, we successfully induced colitisassociated CRC in Balb/c mice. As reported before, in the AOM and DSS-induced CRC, the tumor promoting effect of IL- 6 could be inhibited through treatment with anti-IL-6 receptor antibody (22). Obvious reduced tumor development was also observed in IL-6-/- mice exposed to the AOM and DSS model (23). These previous data together with our results indicated that IL-6 might play a key role in the initiation and progression of colitis-associated CRC. Moreover, blocking the IL-6 function could significantly inhibit the tumor development of CRC.

The IL-6 dependent, signal transducer and activator of transcription-3 (STAT3), the suppressor of cytokine signaling-3 (SOCS3), and the vascular endothelial growth factor receptor-2 (VEGFR2) were reported to be of critical importance for tumor development in the colitis-associated CRC mouse model (23-26). However, only few studies have investigated whether the growth promoting effect of IL-6 in the AOM and DSS colitis-associated CRC mouse model is realized by regulating the aerobic glycolysis. In this study, although key genes involved in aerobic glycolysis were significantly downregulated by anti-IL-6 receptor antibody, plasma concentrations of glucose and lactate were not significantly affected. However, we found the rates of glucose consumption and lactate production were increased by treatment with IL-6 in CRC cells. The differences might be explained by the multiple factors that influence the plasma concentration of glucose and lactate in the whole body.

Genetic disorders have been reported to be related to the phenotypical changes of the morphological progression sequence in the inflammation/adenoma/carcinoma (27). However, whether the aerobic glycolysis is involved in inflammation/adenoma/carcinoma sequential changes is largely unknown. In the present study, we found that key genes of aerobic glycolysis (especially PFKFB3) were significantly downregulated by anti-IL-6 receptor antibody treatment at early stage of CRC. Furthermore, the expression of PFKFB3 was found to be significantly higher in human colorectal adenoma tissue than colorectal inflammation tissue. These results indicated that IL-6 might exert a tumor promoting effect by regulating PFKFB3 starting at the colorectal adenoma stage. However, due to the limited number of human tissues, the expression profile of PFKFB3 upon tumor progression needs future study.
As far as we known, this is the first report on the PFKFB3 expression status in a large human population with 87 sporadic CRC patients showing a close correlation between PFKFB3 expression and several clinicopathological factors. However, whether the PFKFB3 expression can be used as an independed prognostic factor for sporadic CRC patients needs long-term follow-up.

The growth-promoting effect of IL-6 on CRC cells in vitro was reported 3 decades ago (28). In addition, the aerobic glycolysis has been proposed to support the proliferative demands of cancer cells (7). Therefore, we examined whether IL-6 exerts its tumor promoting effect by regulating the aerobic glycolysis in vitro. Results showed that knockdown of PFKFB3 not only reversed the enhanced aerobic glycolysis but also inhibited tumor cell proliferation and migration. The potential mechanisms of how IL-6 upregulates PFKFB3 are that IL-6 increases the expression of HIF-1 and HIF-1 activates transcription of PFKFB3 (29). However, what is the concrete mechanism of how IL-6 exerts its tumor promoting effect by regulating the PFKFB3 in CRC is still under further consideration.

In this study, the inhibitory effect of anti-IL-6 receptor antibody on the development of colitis-associated CRC was observed. In fact, growing evidence supports a critical role for IL-6 signaling during the development of both sporadic and inflammation-associated CRC (5). Therefore, new therapeutic targeting IL-6 pathway might offer a promising option for treatment of CRC patients. To date, anti-IL- 6 receptor antibody has been approved for treatment of chronic inflammatory diseases such as rheumatoid arthritis and juvenile idiopathic arthritis by the Food and Drug Administration (FDA) (30). Although the therapeutic effect of anti-IL-6 receptor antibody on CRC has not been confirmed in clinical trials, anti-IL-6 receptor antibody provides a new possible theoretical strategy for treatment of patients with CRC. Therefore, further study could pay attention to the therapeutic effect of anti-IL-6 receptor antibody on CRC, especially the colitis-associated CRC.

In summary, the present study provides direct evidence that chronic inflammation (IL-6) promotes the initiation and progression of $\mathrm{CRC}$ by regulating aerobic glycolysis (especially the PFKFB3). As anti-IL-6 receptor antibody has a potential clinical usefulness for treatment of $\mathrm{CRC}$, our findings provide theoretical support for such anti-neoplasia strategy.

\section{Acknowledgements}

The present study was supported by the National Natural Science Foundation of China (81372197).

\section{References}

1. Siegel RL, Miller KD and Jemal A: Cancer statistics, 2015. CA Cancer J Clin 65: 5-29, 2015.

2. Sung JJ, Lau JY, Goh KL and Leung WK; Asia Pacific Working Group on Colorectal Cancer: Increasing incidence of colorectal cancer in Asia: Implications for screening. Lancet Oncol 6: 871-876, 2005.

3. Cunningham D, Atkin W, Lenz HJ, Lynch HT, Minsky B Nordlinger B and Starling N: Colorectal cancer. Lancet 375: 1030-1047, 2010

4. Ullman TA and Itzkowitz SH: Intestinal inflammation and cancer. Gastroenterology 140: 1807-1816, 2011. 
5. Waldner MJ, Foersch S and Neurath MF: Interleukin-6--a key regulator of colorectal cancer development. Int J Biol Sci 8: $1248-1253,2012$.

6. Li Y, de Haar C, Chen M, Deuring J, Gerrits MM, Smits R, Xia B, Kuipers EJ and van der Woude CJ: Disease-related expression of the IL6/STAT3/SOCS3 signalling pathway in ulcerative colitis and ulcerative colitis-related carcinogenesis. Gut 59: 227-235, 2010.

7. Vander Heiden MG, Cantley LC and Thompson CB: Understanding the Warburg effect: The metabolic requirements of cell proliferation. Science 324: 1029-1033, 2009.

8. Hsu PP and Sabatini DM: Cancer cell metabolism: Warburg and beyond. Cell 134: 703-707, 2008

9. Hanahan D and Weinberg RA: Hallmarks of cancer: The next generation. Cell 144: 646-674, 2011.

10. Semenza GL: HIF-1: Upstream and downstream of cancer metabolism. Curr Opin Genet Dev 20: 51-56, 2010.

11. Christofk HR, Vander Heiden MG, Harris MH, Ramanathan A, Gerszten RE, Wei R, Fleming MD, Schreiber SL and Cantley LC: The M2 splice isoform of pyruvate kinase is important for cancer metabolism and tumour growth. Nature 452: 230-233, 2008.

12. Ros S and Schulze A: Balancing glycolytic flux: The role of 6-phosphofructo-2-kinase/fructose 2,6-bisphosphatases in cancer metabolism. Cancer Metab 1: 8, 2013.

13. Atsumi T, Chesney J, Metz C, Leng L, Donnelly S, Makita Z, Mitchell R and Bucala R: High expression of inducible 6-phosphofructo-2-kinase/fructose-2,6-bisphosphatase (iPFK-2; PFKFB3) in human cancers. Cancer Res 62: 5881-5887, 2002.

14. Obach M, Navarro-Sabaté A, Caro J, Kong X, Duran J, Gómez M, Perales JC, Ventura F, Rosa JL and Bartrons R: 6-Phosphofructo2-kinase (pfkfb3) gene promoter contains hypoxia-inducible factor-1 binding sites necessary for transactivation in response to hypoxia. J Biol Chem 279: 53562-53570, 2004.

15. Neufert C, Becker $C$ and Neurath MF: An inducible mouse model of colon carcinogenesis for the analysis of sporadic and inflammation-driven tumor progression. Nat Protoc 2: 1998-2004, 2007.

16. Kawauchi K, Araki K, Tobiume K and Tanaka N: p53 regulates glucose metabolism through an IKK-NF-kappaB pathway and inhibits cell transformation. Nat Cell Biol 10: 611-618, 2008.

17. Yuan L, Han J, Meng Q, Xi Q, Zhuang Q, Jiang Y, Han Y, Zhang B, Fang J and Wu G: Muscle-specific E3 ubiquitin ligases are involved in muscle atrophy of cancer cachexia: An in vitro and in vivo study. Oncol Rep 33: 2261-2268, 2015.

18. Grivennikov SI and Karin M: Inflammation and oncogenesis: A vicious connection. Curr Opin Genet Dev 20: 65-71, 2010.

19. Takahashi M, Nakatsugi S, Sugimura T and Wakabayashi K: Frequent mutations of the beta-catenin gene in mouse colon tumors induced by azoxymethane. Carcinogenesis 21: 1117-1120, 2000 .
20. Wang QS, Papanikolaou A, Sabourin CL and Rosenberg DW: Altered expression of cyclin D1 and cyclin-dependent kinase 4 in azoxymethane-induced mouse colon tumorigenesis. Carcinogenesis 19: 2001-2006, 1998

21. Suzuki R, Kohno H, Sugie S, Nakagama H and Tanaka T: Strain differences in the susceptibility to azoxymethane and dextran sodium sulfate-induced colon carcinogenesis in mice. Carcinogenesis 27: 162-169, 2006.

22. Becker C, Fantini MC, Schramm C, Lehr HA, Wirtz S, Nikolaev A, Burg J, Strand S, Kiesslich R, Huber S, et al: TGF-beta suppresses tumor progression in colon cancer by inhibition of IL-6 trans-signaling. Immunity 21: 491-501, 2004.

23. Grivennikov S, Karin E, Terzic J, Mucida D, Yu GY, Vallabhapurapu S, Scheller J, Rose-John S, Cheroutre H, Eckmann L, et al: IL-6 and Stat 3 are required for survival of intestinal epithelial cells and development of colitis-associated cancer. Cancer Cell 15: 103-113, 2009.

24. Bollrath J, Phesse TJ, von Burstin VA, Putoczki T, Bennecke M, Bateman T, Nebelsiek T, Lundgren-May T, Canli O, Schwitalla S, et al: gp130-mediated Stat3 activation in enterocytes regulates cell survival and cell-cycle progression during colitis-associated tumorigenesis. Cancer Cell 15: 91-102, 2009.

25. Rigby RJ, Simmons JG, Greenhalgh CJ, Alexander WS and Lund PK: Suppressor of cytokine signaling 3 (SOCS3) limits damage-induced crypt hyper-proliferation and inflammationassociated tumorigenesis in the colon. Oncogene 26: 4833-4841, 2007.

26. Waldner MJ, Wirtz S, Jefremow A, Warntjen M, Neufert C, Atreya R, Becker C, Weigmann B, Vieth M, Rose-John S, et al: VEGF receptor signaling links inflammation and tumorigenesis in colitis-associated cancer. J Exp Med 207: 2855-2868, 2010.

27. Arvelo F, Sojo F and Cotte C: Biology of colorectal cancer. E Cancer Medical Sci 9: 520, 2015.

28. Lahm H, Petral-Malec D, Yilmaz-Ceyhan A, Fischer JR, Lorenzoni M, Givel JC and Odartchenko N: Growth stimulation of a human colorectal carcinoma cell line by interleukin-1 and -6 and antagonistic effects of transforming growth factor beta 1 . Eur J Cancer 28A: 1894-1899, 1992.

29. Lang SA, Moser C, Gaumann A, Klein D, Glockzin G, Popp FC, Dahlke MH, Piso P, Schlitt HJ, Geissler EK, et al: Targeting heat shock protein 90 in pancreatic cancer impairs insulin-like growth factor-I receptor signaling, disrupts an interleukin-6/ signal-transducer and activator of transcription 3/hypoxiainducible factor-1alpha autocrine loop, and reduces orthotopic tumor growth. Clin Cancer Res 13: 6459-6468, 2007.

30. Tanaka T, Narazaki M and Kishimoto T: Therapeutic targeting of the interleukin-6 receptor. Annu Rev Pharmacol Toxicol 52: 199-219, 2012. 